Supporting Information

\title{
Colloidal Gels for Guiding Endothelial Cell Organization via Microstructural Morphology
}

\author{
Yuan Yuan ${ }^{\mathrm{a}}$, Sukanya Basub ${ }^{\mathrm{b}}$, Meng Huisan Lin ${ }^{\mathrm{a}}$ Shruti Shukla ${ }^{\mathrm{a}}$, Debanjan Sarkar,c,* \\ aDepartment of Biomedical Engineering, University at Buffalo, The State University of New \\ York, Buffalo, NY 14260, USA \\ bepartment of Pharmacology and Therapeutics, Roswell Park Comprehensive Cancer Center, \\ Buffalo, NY 14263, USA \\ ${ }^{\mathrm{c}}$ Department of Chemical and Biological Engineering, University at Buffalo, The State \\ University of New York, Buffalo, NY 14260, USA
}

*Correspondence to: D. Sarkar.

Biomedical Engineering, University at Buffalo, Ph: 716-645-8497, Fax: 716-645-2207

Email: debanjan@buffalo.edu 
Figure S1:

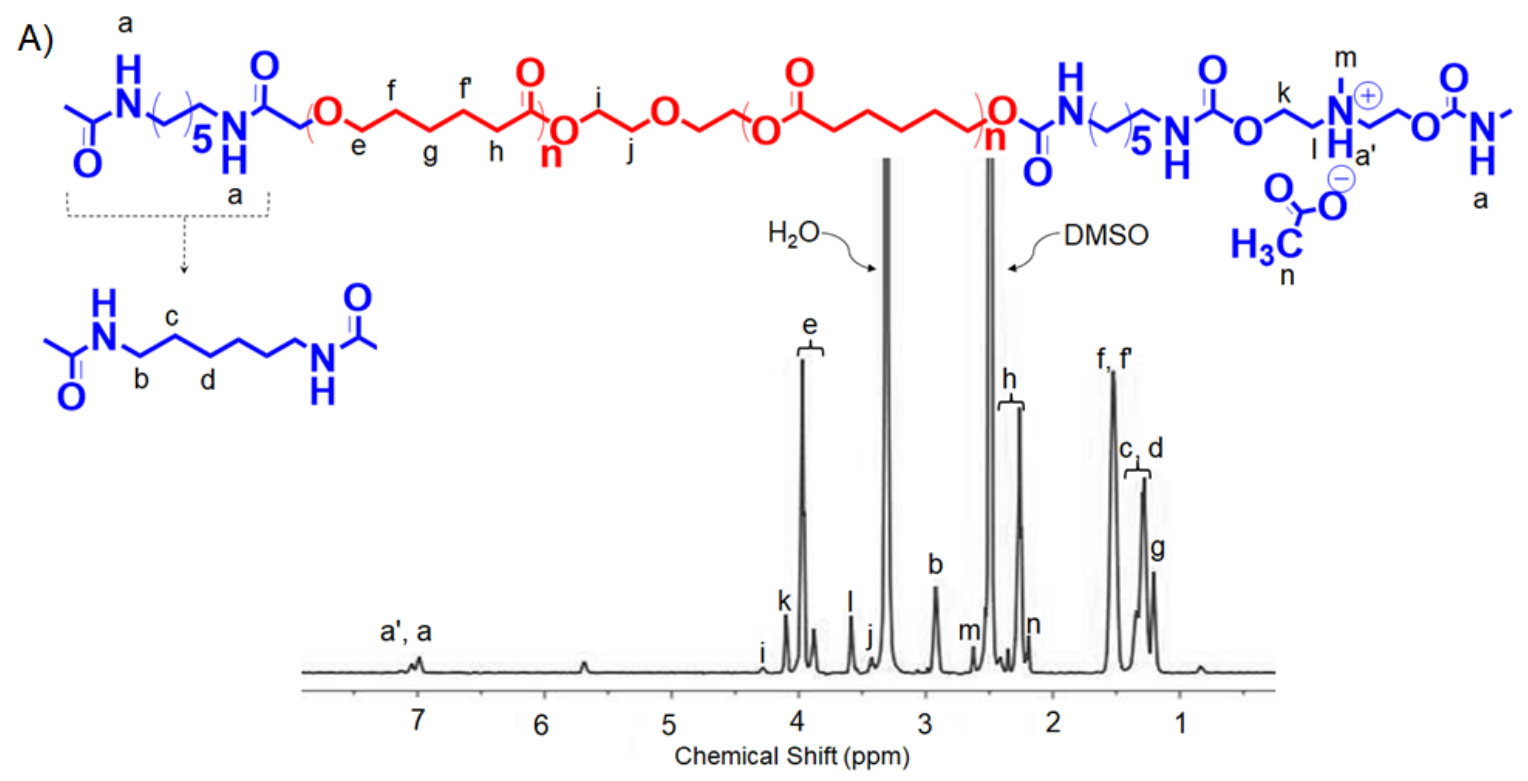

B)

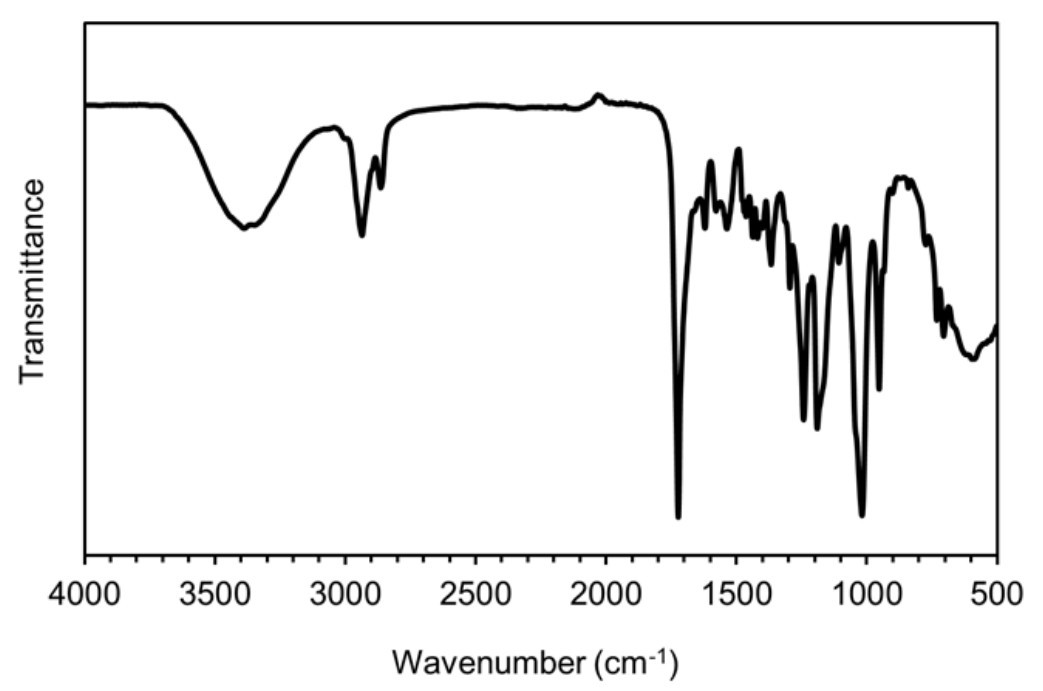

Figure S1: A) Molecular structure of polycaprolactone based cationic polyurethane. Structural analysis with ${ }^{1} \mathrm{H}$ NMR (500 MHz, DMSOd 6 ) with peak assignments showing structure of the polyurethane. B) FTIR analysis of the polyurethane with ester functionality of polycaprolactone segment $\left(\sim 1720 \mathrm{~cm}^{-1}\right)$ and $-\mathrm{NH}$ functionality of urethane links $\left(\sim 3400 \mathrm{~cm}^{-1}\right)$. 
Figure S2:
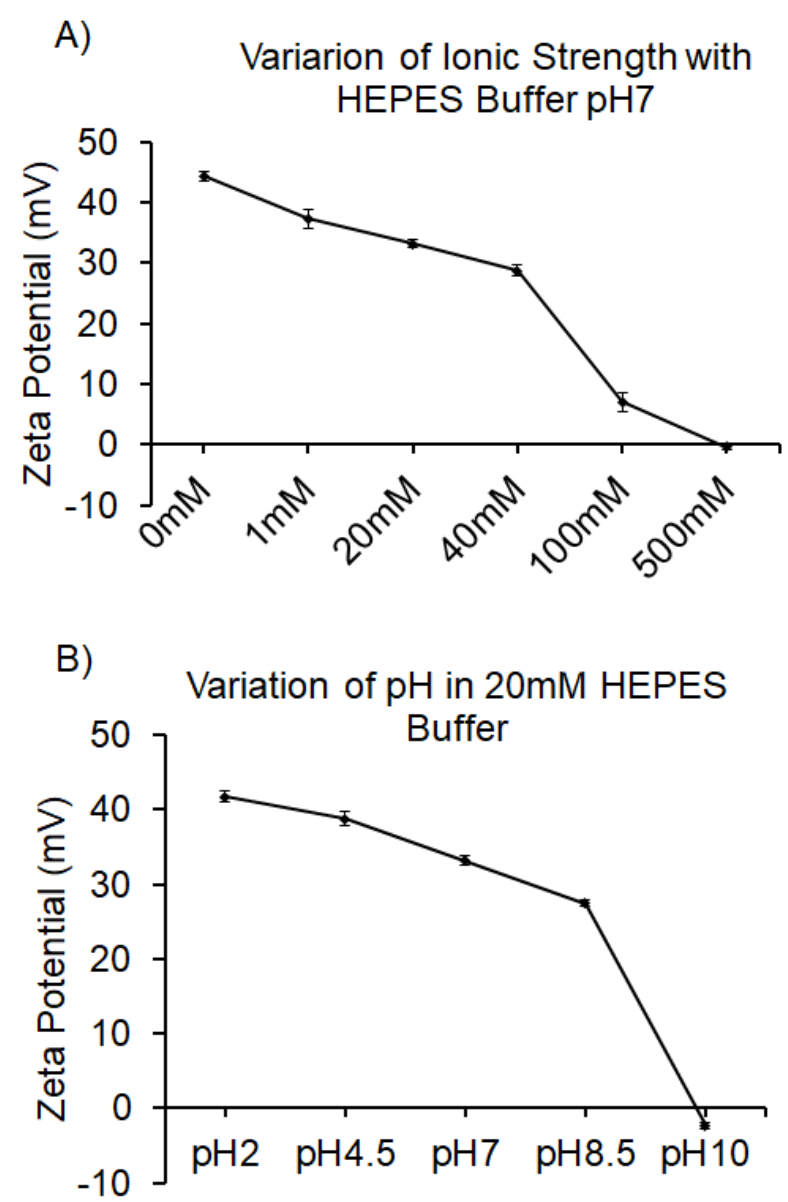

Figure S2: Variation of zeta potential of cationic PU colloidal particles with (A) increasing ionic strength at constant $\mathrm{pH}$, and (B) increasing $\mathrm{pH}$ at constant ionic strength, shows presence positive charge on cationic PU colloidal particles. 
Figure S3:

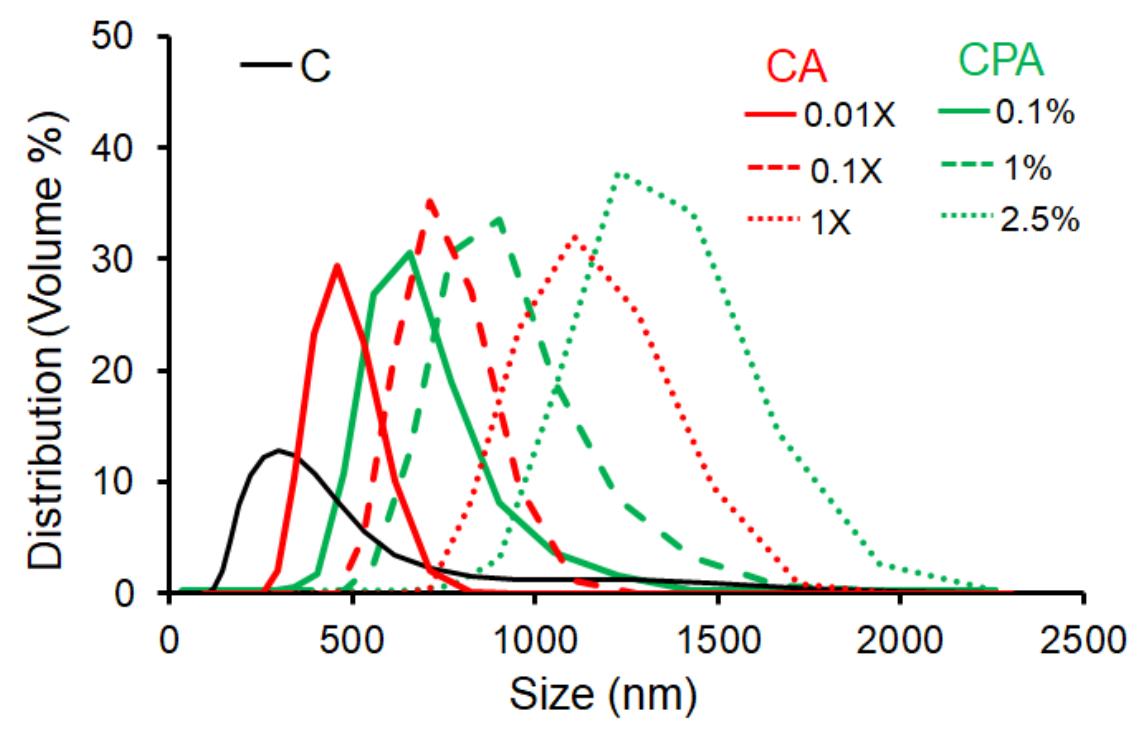

Figure S3: Size distribution of cationic PU colloidal particles and aggregated colloidal particles with different strength of phosphate buffer saline (CA) and Na-salt of polyacrylic acid (CPA).

Figure S4:

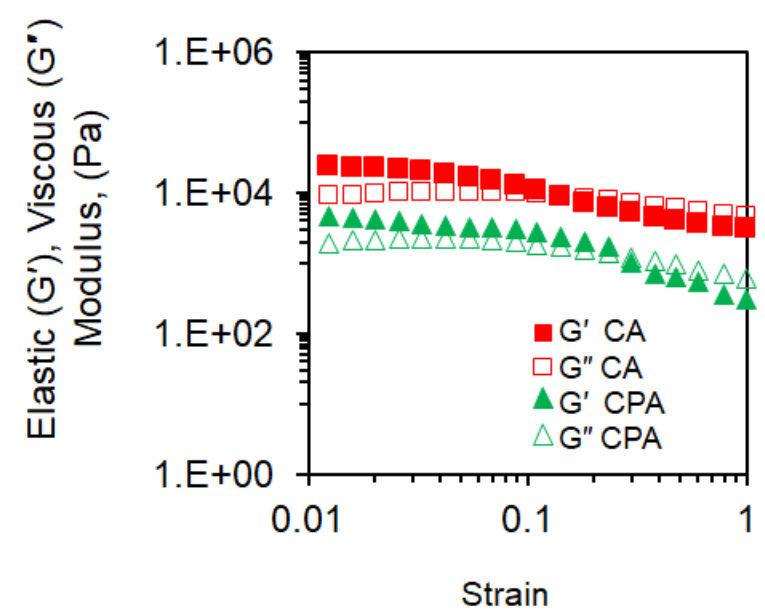

Figure S4: Oscillatory amplitude sweep at constant frequency of CA and CPA colloidal gels prepared from 0.1 particle fraction. 
Figure S5:

A)
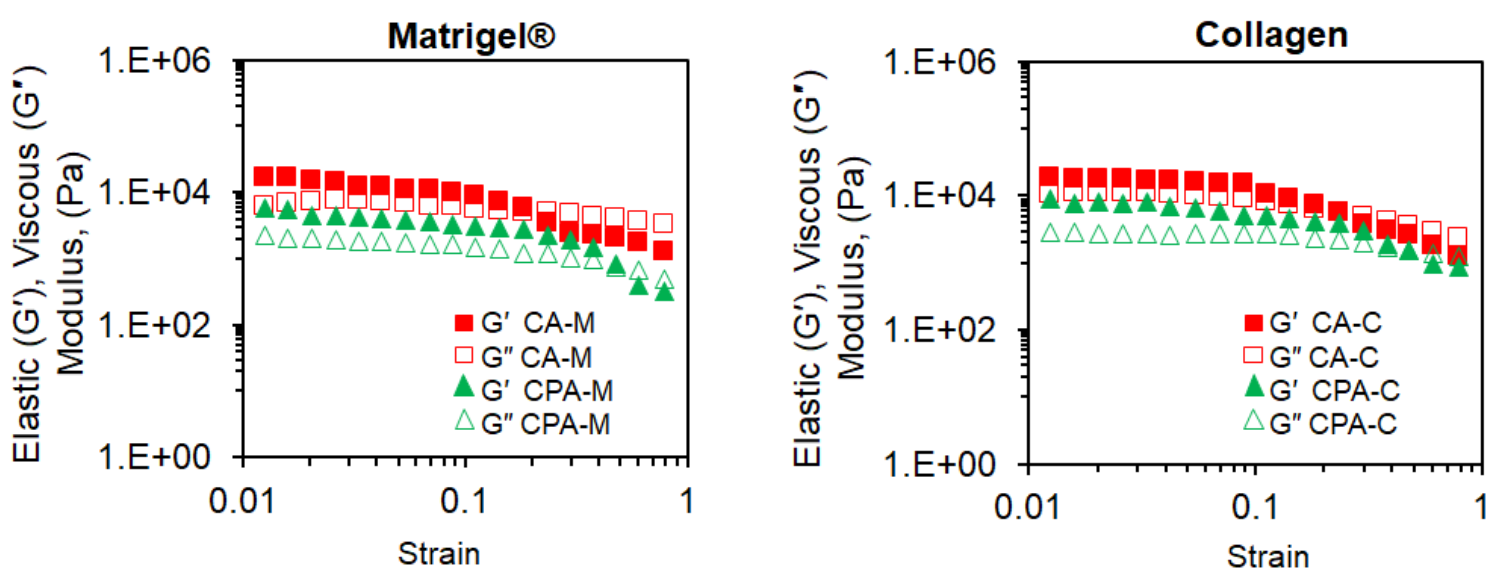

B)
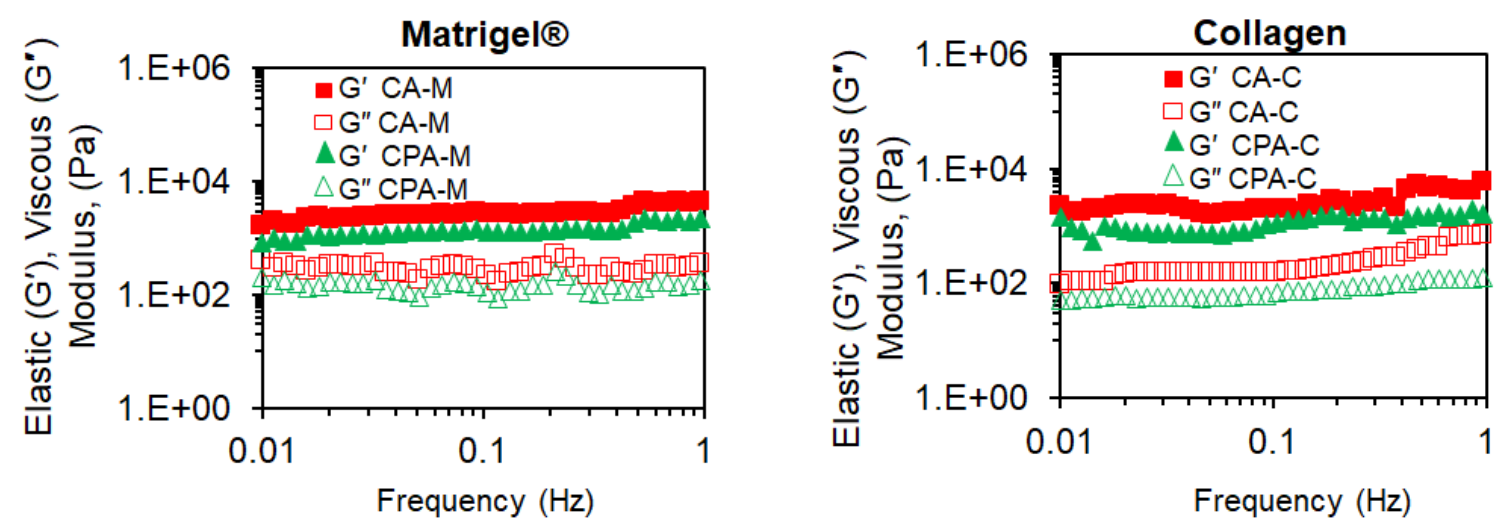

Figure S5: Mechanical characterizations of composite colloidal gel from rheology. A) Oscillatory strain amplitude sweep measured at constant frequency. C) Oscillatory frequency sweep at constant strain for matrigel and collagen based composite gels for CA and CPA prepared from 0.1 particle fraction. 
Figure S6:

A)
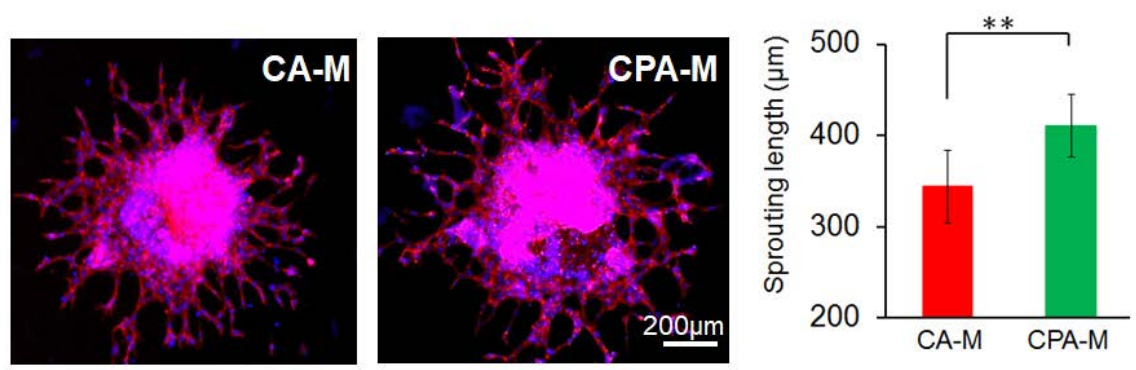

B)
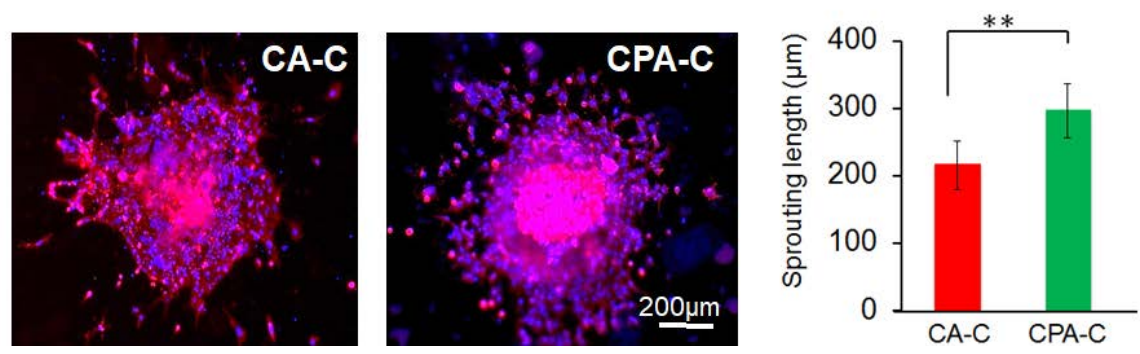

Figure S6: Emigration (sprouting) of ECs from EC spheroid in colloidal aggregate embedded endothelial matrix. A) Sprouting of ECs in CA and CPA aggregate embedded in Matrigel (CA-M and CPA-M) and the length of sprouted ECs at 48 hrs. B) Sprouting of ECs in CA and CPA aggregate embedded in collagen I (CA-C and CPA-C) and the length of sprouted ECs at 48 hrs.

Figure S7:

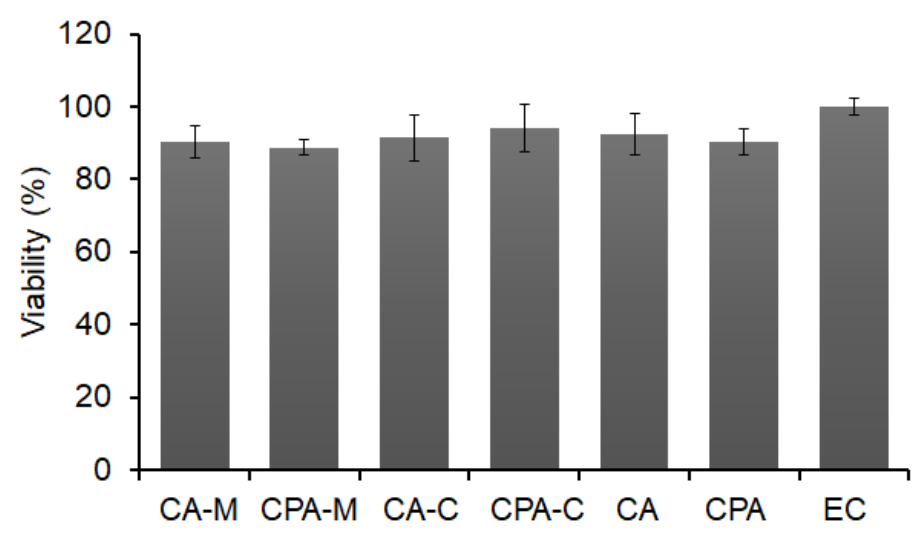

Figure S7: Viability of ECs in native CA and CPA colloidal gels and composite colloidal gels with Matrigel and collagen measured at 48 hrs showing no significant adverse effect of gel matrices on cell viability (compared to cell only control group EC). 
Figure S8:

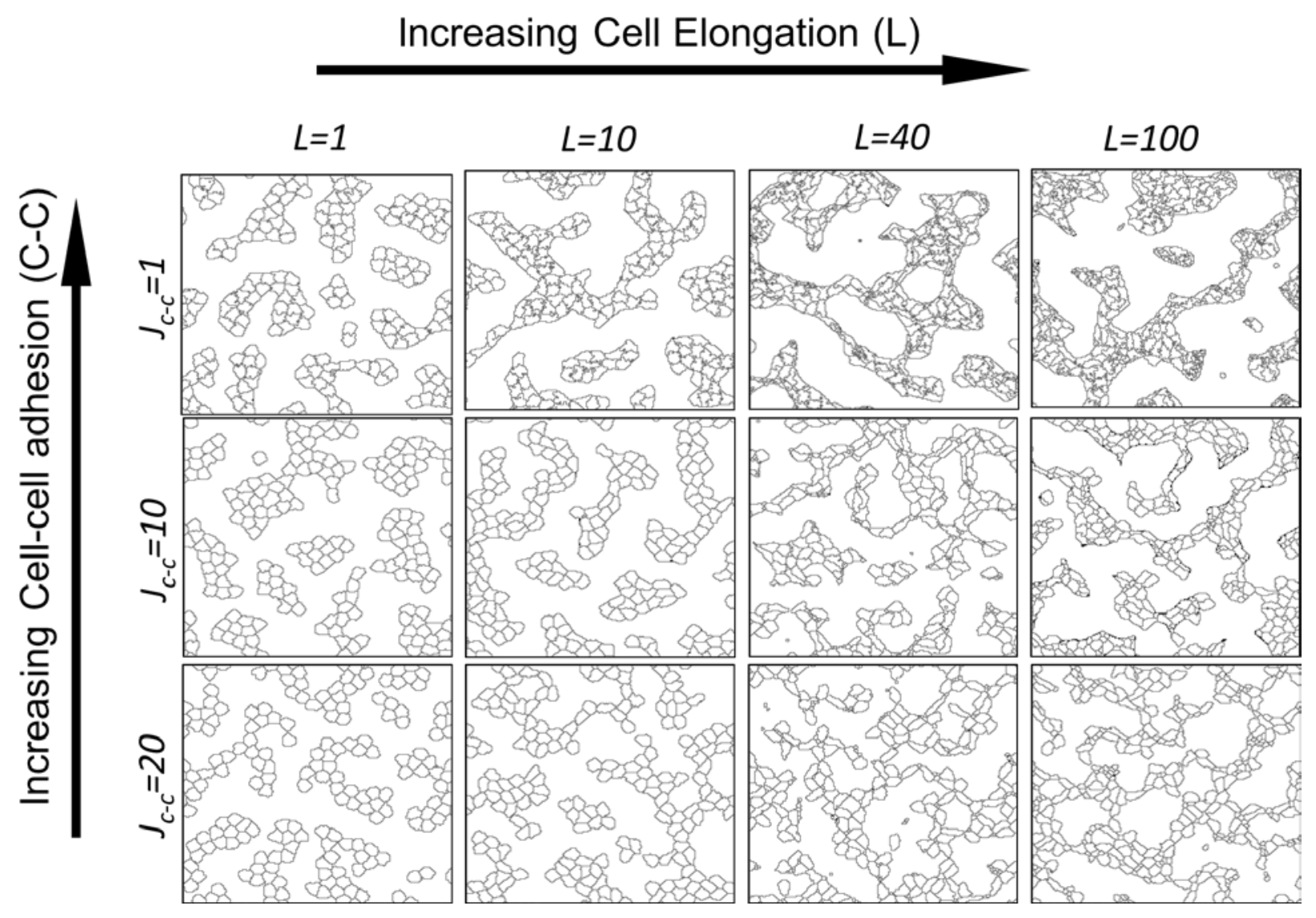

Figure S8: Simulated vascular patterns obtained from combinatorial analysis of cell-cell (C-C) adhesion and cell elongation (L) with systematic change in $J_{c-c}$ (higher value indicates low cellcell adhesion) and $L$ (larger value indicates increased cell elongation) respectively, under low chemotactic strength. 
Figure S9:

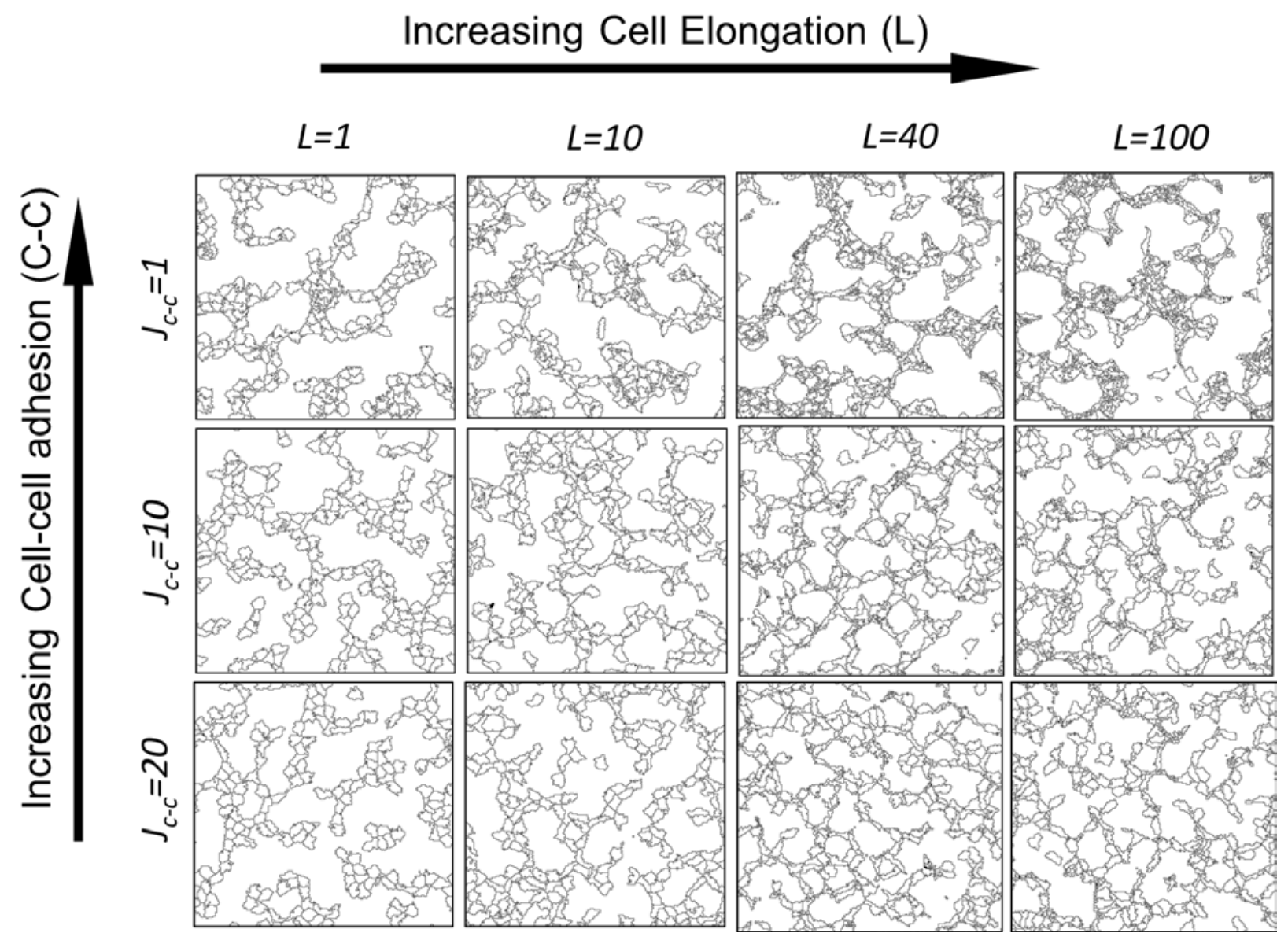

Figure S9: Simulated vascular patterns obtained from combinatorial analysis of cell-cell (C-C) adhesion and cell elongation (L) with systematic change in $J_{c-c}$ (higher value indicates low cellcell adhesion) and $L$ (larger value indicates increased cell elongation) respectively, under high chemotactic strength. 
Fig. S10-S15: Additional images from independent sets of experiments
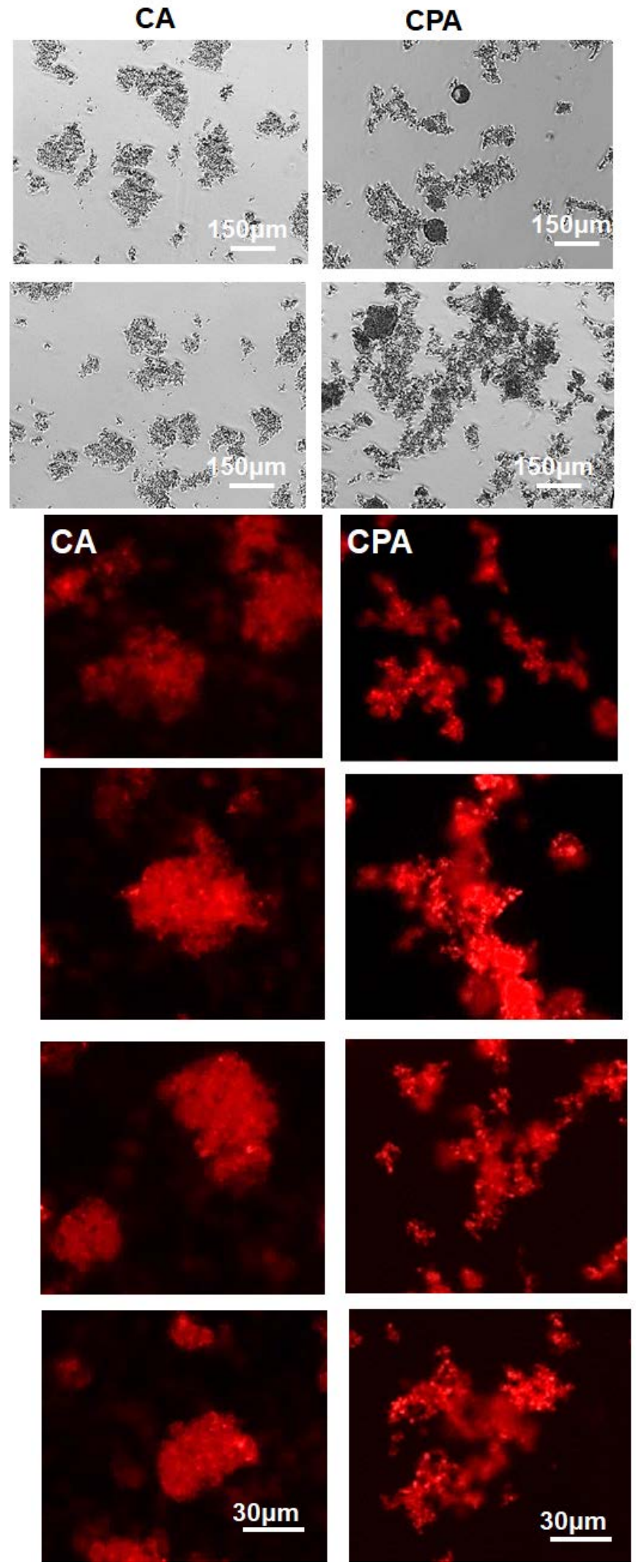

Figure S10: Additional images from independent experiments corresponding to Figure 2. Brightfield image of CA and CPA colloidal aggregates from two additional independent sets of experiment. Fluorescent microscopic images of CA and CPA colloidal aggregates from four additional independent sets of experiment. 

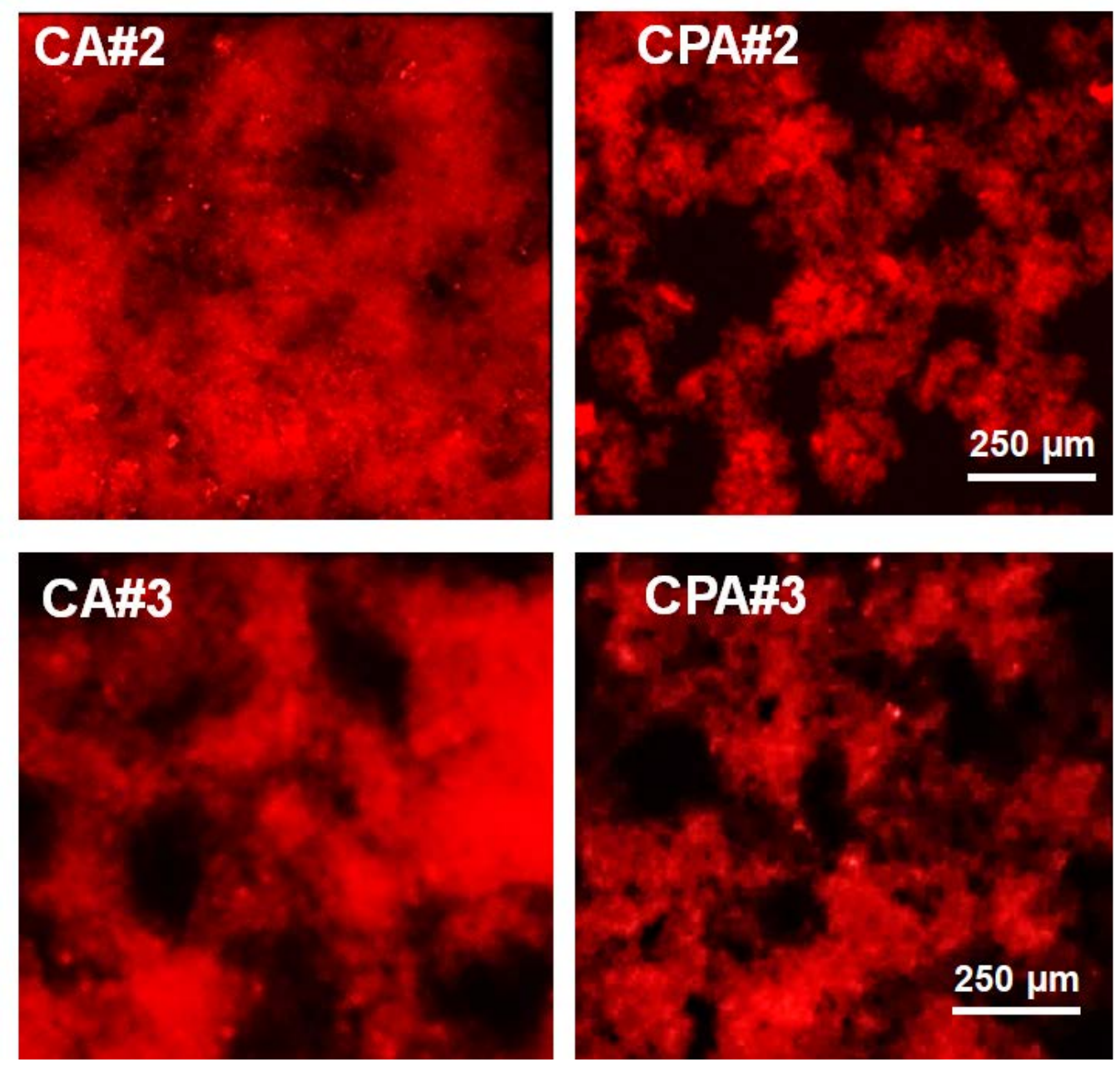

Figure S11: Additional images from independent experiments corresponding to Figure 3 related to fluorescent images and 3D interactive surface plot (color code indicates z-depth) of CA and CPA gels for analysis of morphology and spatial distribution of voids in the gels from two additional independent sets of experiment. 

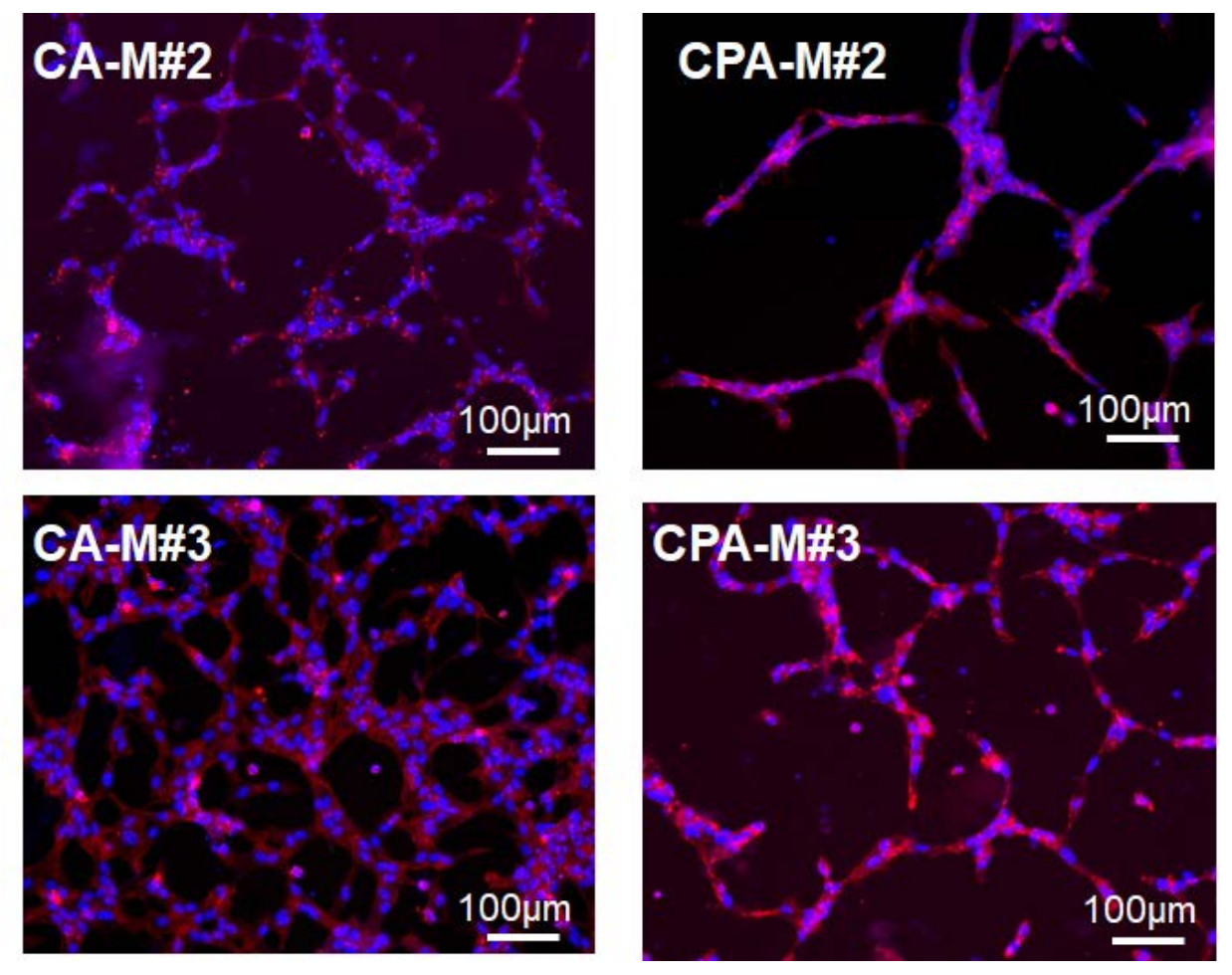

Figure S12: Additional images from independent experiments corresponding to Figure 5A related to fluorescent images of EC organization (red=actin and blue=nucleus) in CA and CPA at 0.1 particulate fraction embedded in Matrigel (CA-M and CPA-M) from two additional independent sets of experiment. 

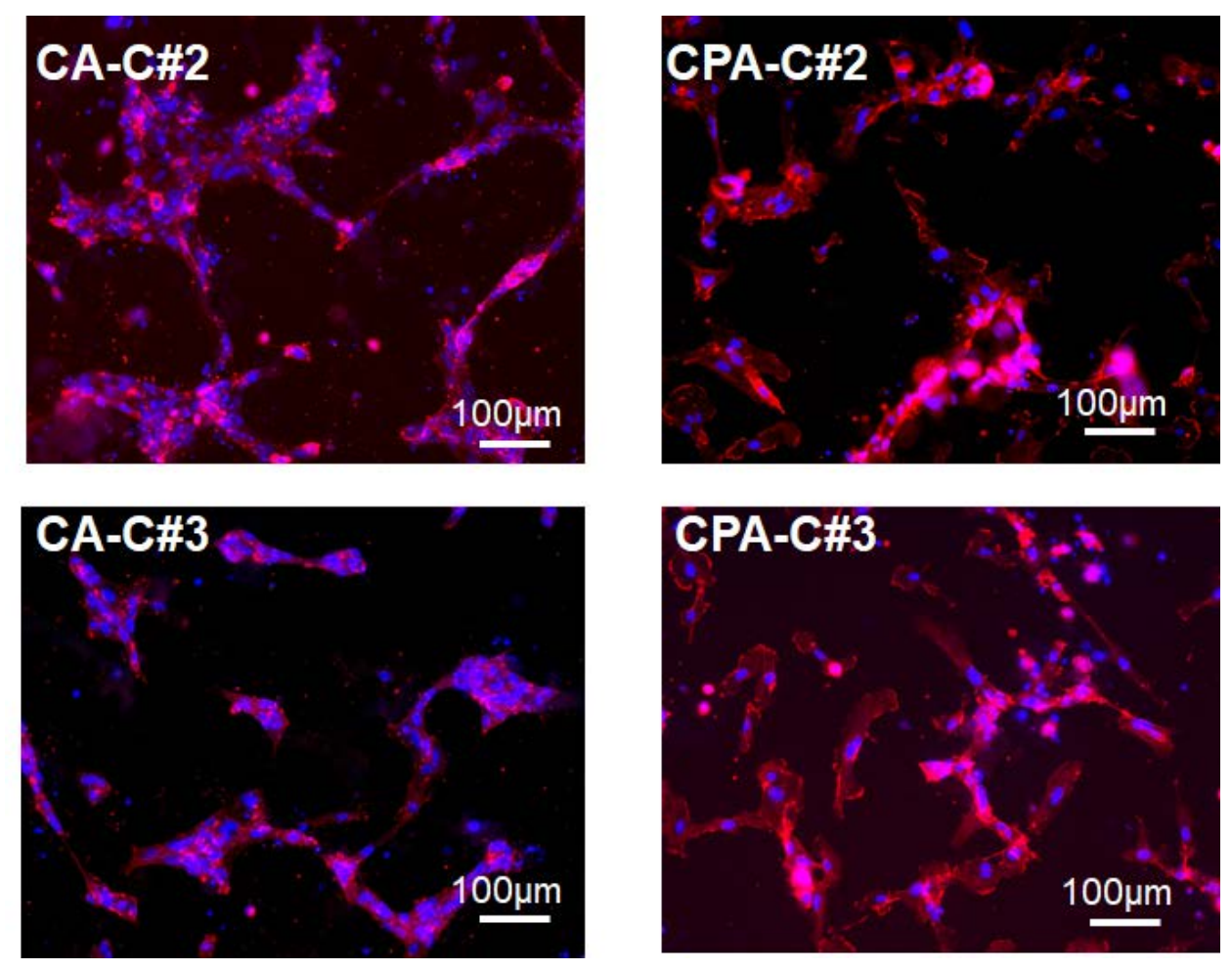

Figure S13: Additional images from independent experiments corresponding to Figure 5C related to fluorescent images of EC organization (red=actin and blue=nucleus) in CA and CPA at 0.1 particulate fraction embedded in Collagen-I (CA-C and CPA-C) from two additional independent sets of experiment. 

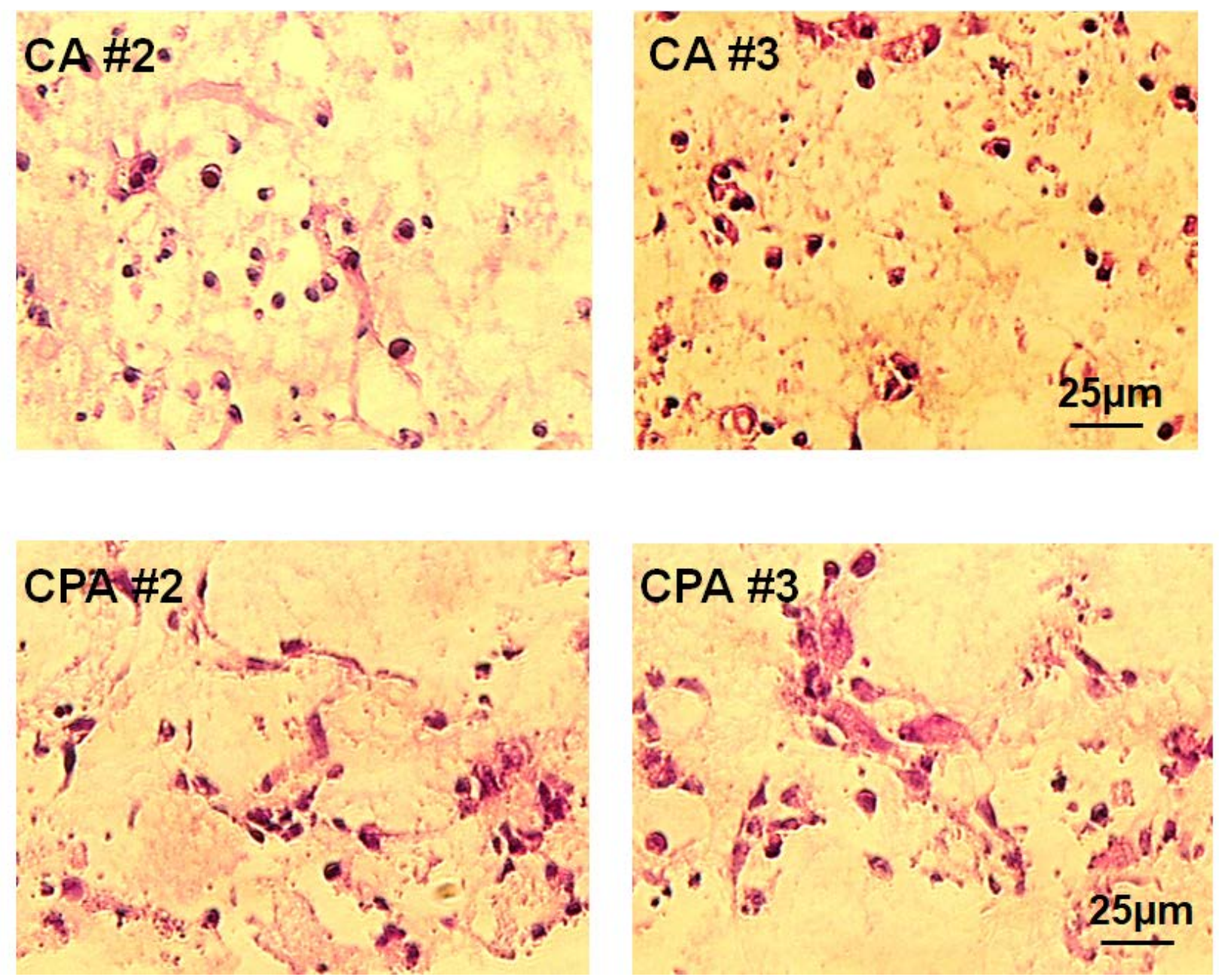

Figure S14: Additional images from independent experiments corresponding to Figure 6A related to H\&E stained images of ECs in CA and CPA colloidal gel prepared from 0.1 particle fraction at $48 \mathrm{hrs}$ from two additional independent sets of experiment. 

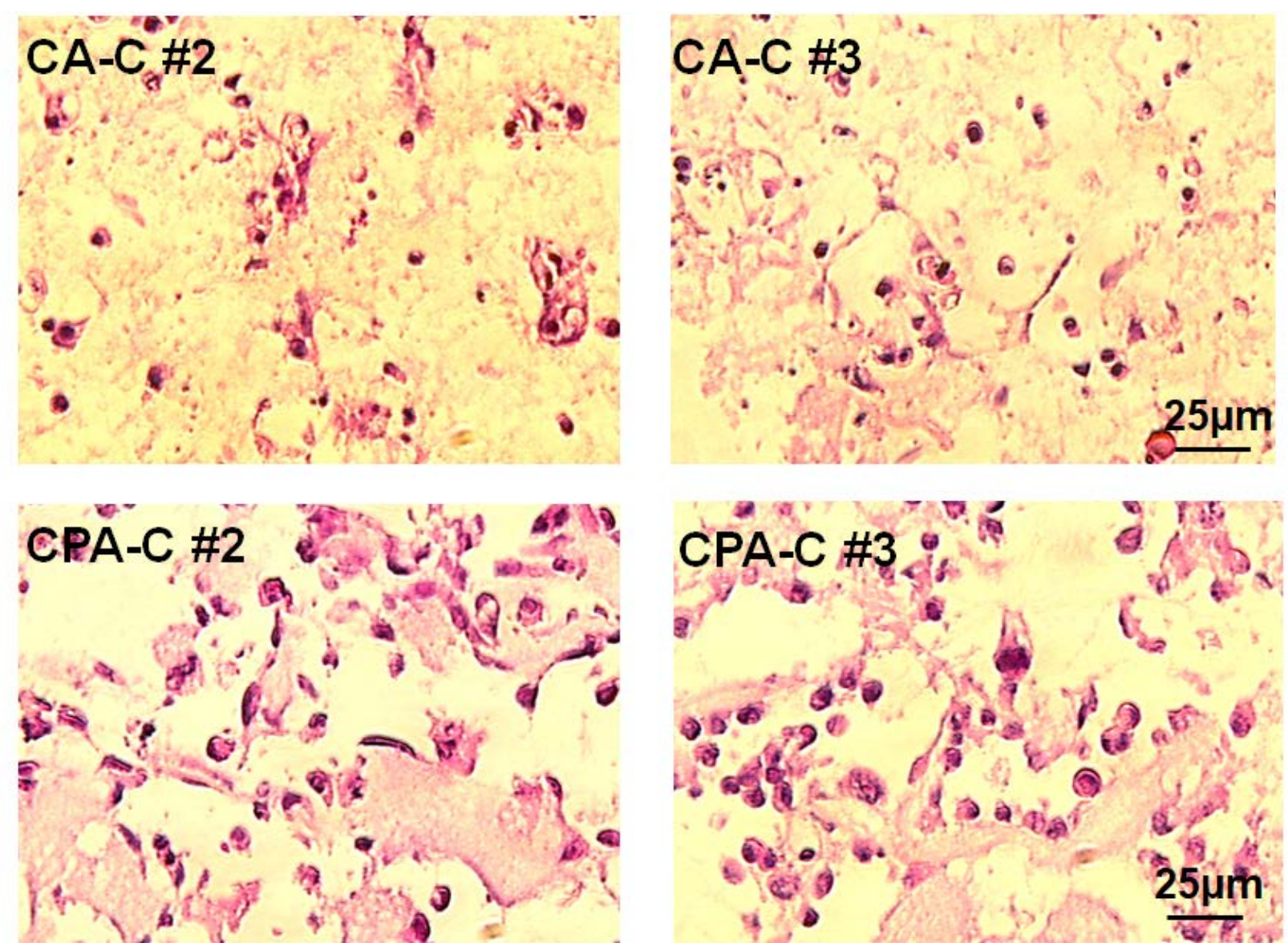

Figure S15: Additional images from independent experiments corresponding to Figure 6C related H\&E stained images of ECs in composite CA and CPA colloidal gel prepared from 0.1 particle fraction in presence of collagen I (CA-C and CPA-C) at 48 hrs from two additional independent sets of experiment. 

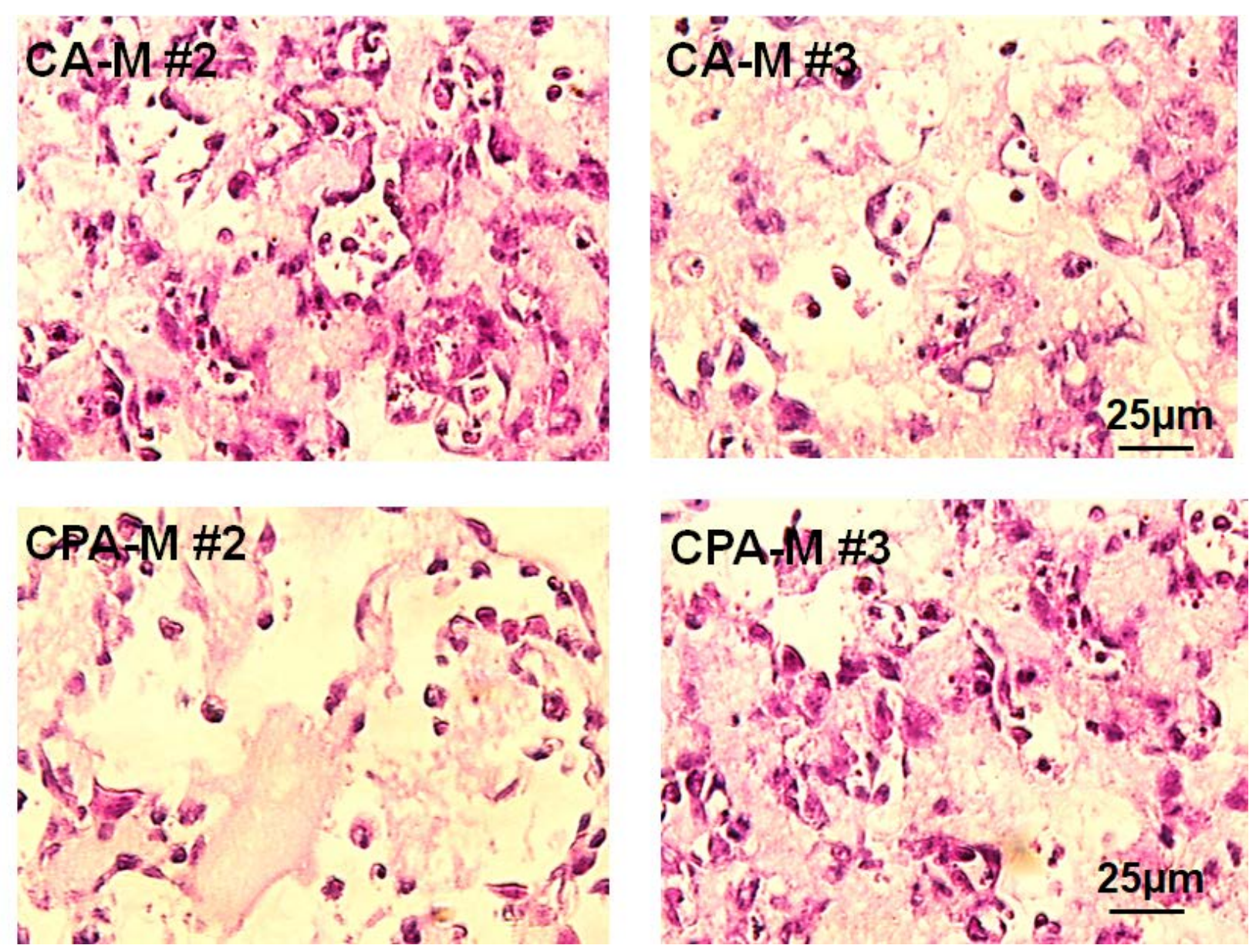

Figure S16: Additional images from independent experiments corresponding to Figure 6C related H\&E stained images of ECs in composite CA and CPA colloidal gel prepared from 0.1 particle fraction in presence of Matrigel (CA-M and CPA-M) at 48 hrs from two additional independent sets of experiment. 